\title{
Mesoscopic structuring and yield stress of magnetofluidized fine particles
}

\author{
J. M. Valverde ${ }^{1(a)}$, M. J. Espin ${ }^{2}$, M. A. S. Quintanilla ${ }^{1}$ and A. Castellanos ${ }^{1}$ \\ ${ }^{1}$ Department of Electronics and Electromagnetism, University of Seville - Avda. Reina Mercedes s/n, \\ 41012 Seville, Spain, EU \\ ${ }^{2}$ Department of Applied Physics II, University of Seville - Avda. Reina Mercedes s/n, 41012 Seville, Spain, EU
}

received 1 September 2009; accepted 6 October 2009

published online 10 November 2009

PACS 47.55.Lm - Fluidized beds

PACS 47.55.Kf - Particle-laden flows

PACS $47.65 . \mathrm{Cb}$ - Magnetic fluids and ferrofluids

\begin{abstract}
The fluidization behavior of a bed of fine magnetizable particles excited by an externally applied magnetic field is found to depend on the aggregative nature of the particles before the field was applied. Usually nonaggregated particles organize in quasivertical local linear chains when the field is applied. In contrast, naturally aggregated particles form large-scale branched structures when magnetized by an external field. As a consequence the yield stress of magnetically stabilized beds of naturally aggregated particles is relatively increased and the bed can be stabilized at smaller field intensities. As expected from the magnetic cohesive force between magnetized particles, the yield stress is proportional to the square of the magnetic-field intensity, with a proportionality constant that depends on the mesoscopic organization on the magnetic particles. Remarkably, it is found that quasivertical chainlike structures are stable in spite of the fact that the magnetic field is applied in the horizontal direction.
\end{abstract}

Copyright (C) EPLA, 2009

Gas fluidization is a widely used process in the chemical industry to achieve high solid-gas contact efficiency. A fluidized bed typically consists of a particle-laden vessel, with the particle bed kept fluidized by feeding a gas flow through it. As the superficial gas velocity $v_{g}$ is increased there comes a point at which the gas pressure drop balances the material weight per unit area. At this point the particles become fluidized in a usually heterogeneous state, which is characterized by the development of large gas bubbles rising across the bed. Bubbles serve as channels for the gas to bypass the particles, which hampers solid-gas contact efficiency. Externally applied magnetic fields may suppress bubbles when fluidizing magnetic particles [1]. Magnetically stabilized beds yield high solid-gas contact efficiency as a result of their large porosity, which makes them specially suitable for the adsorption of contaminants from a gas stream. Other relevant application of magnetically stabilized beds is their use as magnetically controlled valves. As reviewed by Siegell [2] the earliest report of magnetically stabilized beds dates back to 1960 and was due to Filippov, who

(a) E-mail: jmillan@us.es observed the behavior of a bubbling bed of iron beads subjected to an imposed magnetic field in the vertical direction. Filippov noticed a decrease in particle movement as the strength of the magnetic field was increased until the bed finally reached a stable, calm state. Rosensweig [1] developed a linear stability analysis that was able to predict that the state of fluidization of magnetic particles could be stabilized by the action of a vertical magnetic field. Rosensweig assumed the medium to be inviscid, which in most cases is a drastic idealization of the rheological behavior of magnetically stabilized beds, driving him to less than reliable predictions [1]. Usually, magnetized particles are modeled as point dipoles oriented along the field lines, resulting on chain-like field oriented structures [3]. Magnetofluidized beds excited by a horizontal field should be therefore inherently unstable due to the repulsive force between the chains expected to be oriented in the horizontal direction. However, no experimental evidence for negative cohesion has been ever reported in beds of magnetizable particles [3] and empirical observations have demonstrated that magnetic stabilization is possible for different orientations of the magnetic field, including the horizontal configuration [1]. 
The physical mechanism responsible for the stabilizing cohesive stress of magnetizable fluidized beds remains to be understood.

Flow structuring of magnetizable particles is also of interest to geological studies. Measuring the anisotropy of magnetic susceptibility due to flow alignment of magnetic particles is a method routinely used by geologists to identify palaeoflow directions in volcanic rocks and sediments [4]. In the presence of a magnetic field, the sedimentary magnetic anisotropy should be influenced by the effect of the magnetic field on the process of sedimentation. In spite of its unquestionable role, it is recognized that this effect has been mostly overlooked, which possibly leads to erroneous interpretation of data on magnetic anisotropy. In the nanoworld, controlling the organization of magnetizable nanoparticles on a mesoscopic scale has opened a new area of research to achieve structures for a wide variety of applications. Recently, a coupling between van der Waals and dipolar interparticle interactions has been reported for magnetic nanoparticles dispersed in solution. Aggregates of nanoparticles, formed due to enhanced van der Waals forces, exhibit large dipole moments compared to single nanoparticles, which explains the observation of chain-like mesoscopic structures in the former case when a strong magnetic field is applied [5]. Currently, advanced techniques to capture and store greenhouse gases emissions are being investigated based on the development of tailored nanoparticle adsorbents. Enhanced fluidization of active nanoparticles excited by an external field should increase the solid-gas contact efficiency and therefore improve the adsorption rate of contaminants. The analysis of mesoscopic flow structures of magnetizable particles is thus a matter of interdisciplinary interest in a wide range of scales. In the present work we report observations on magnetofluidized beds of fine magnetizable particles stabilized by a horizontal magnetic field. The powders employed have a distinct aggregative nature in the demagnetized state, which affects their response to a magnetizing field. Results show that at marginal stability the bed is provided with a cohesive stress that can be correlated to magnetic-field strength and mesoscopic structuring of the particles. Moreover, analysis of the field distribution within contacting particles shows that the point dipole approximation is unrealistic since quasivertical chains can be stabilized by a horizontally applied field.

In our experimental setup, the powder bed sample is held in a vertically oriented cylindrical vessel $(2.54 \mathrm{~cm}$ internal diameter) and rests on a porous plate that acts as gas distributor ( $5 \mu \mathrm{m}$ pore size). A controlled flow of filtered and dried air is pumped through the powder bed while the gas pressure drop across it is read from a differential pressure transducer. The powders used in the experiments are demagnetized magnetite and steel powders. Flow properties of these powders have been previously studied by a statistical analysis of avalanches in a half-filled and slowly rotated drum [6]. A relevant
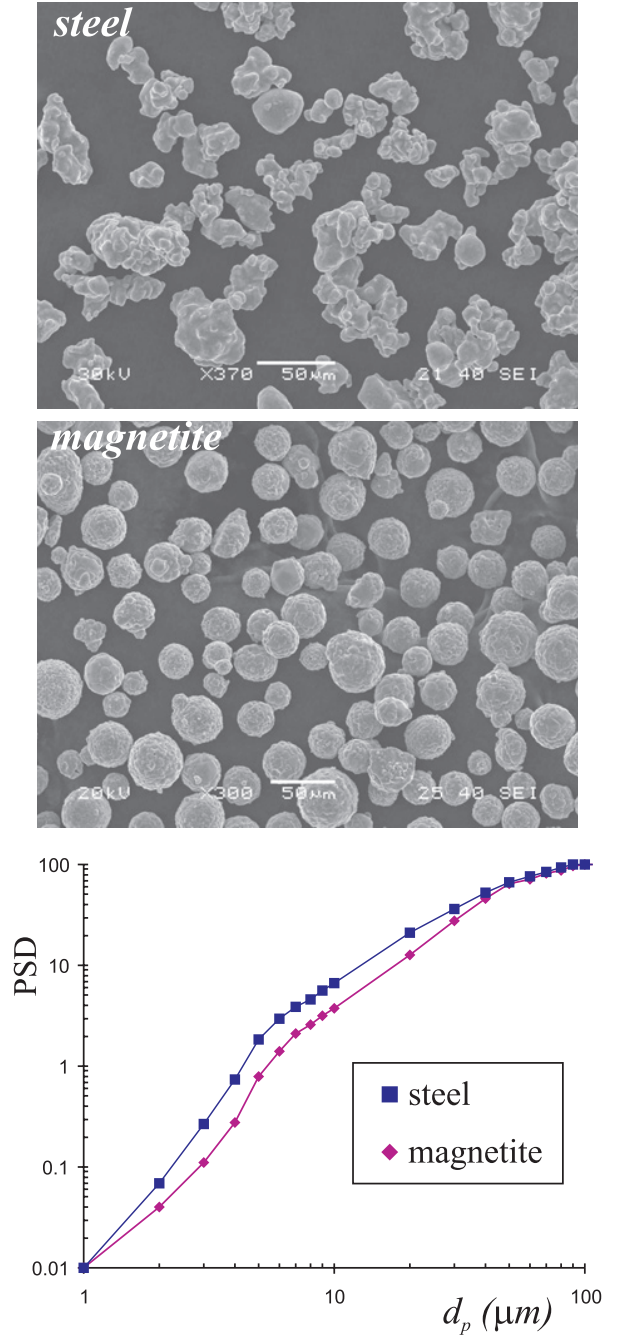

Fig. 1: (Colour on-line) SEM photographs and cumulative particle size distribution of the steel and magnetite particles used in the magnetofluidization experiments.

difference in their flow behavior concerns the distributions of angle of avalanche and angle of repose, which for the steel powder are wider and shifted to higher values, indicating a more cohesive behavior [6]. SEM photographs of both powders can be seen in fig. 1. Steel particles are irregularly shaped and usually form aggregates, in accordance with their higher cohesiveness. In contrast, magnetite particles are roundly shaped and are almost individually dispersed. In fig. 1 the cumulative size distribution of both powders are plotted, which were obtained by means of Galai Cis-1 laser system technique. It is seen a higher presence of fines in the steel powder, which explains their natural aggregative behavior. For particle sizes below $\sim 10 \mu \mathrm{m}$ the attractive van der Waals force becomes several orders of magnitude larger than particle weight, thus enhancing particle aggregation [7]. Our magnetizable powders have been tested as affected by a uniform magnetic field applied in the horizontal direction. The strength of the magnetic 


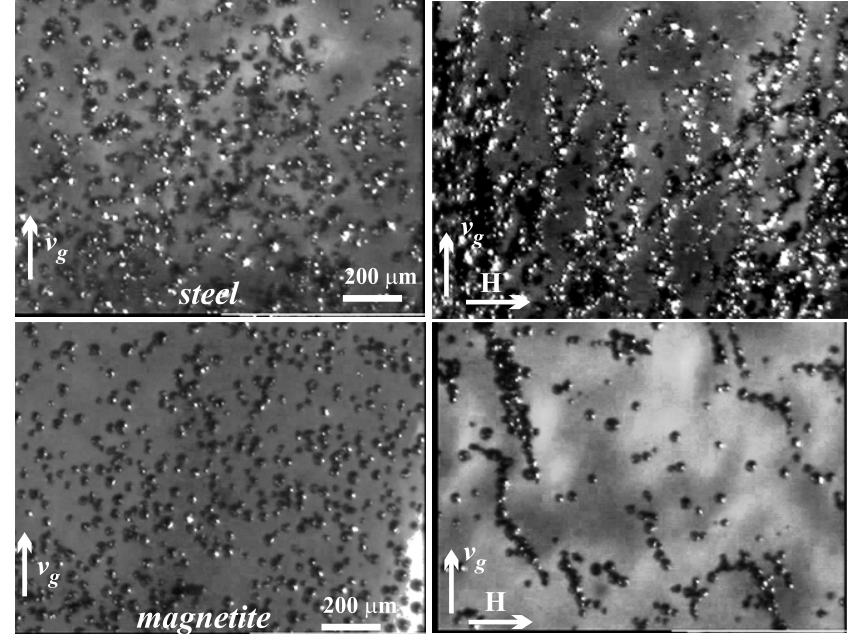

Fig. 2: Optical-microscope images of the steel and magnetite particles in a bed fluidized by a vertical gas flow in the absence (left) and presence (right) of a horizontal magnetic field of strength $H \simeq 4 \mathrm{kA} / \mathrm{m}$.

field is varied by adjusting the electrical current through a pair of square Helmholtz coils and measured by an axial probe. Following the procedure described in [8], the measured bulk susceptibility $\chi$ is similar for both powders $(\chi \simeq 1.8)$.

Visualization of particle arrangement in the fluidized bed as affected by the presence of the horizontal magnetic field was made by carefully lowering edgewise a piece of adhesive tape into the magnetofluidized bed so as to cleave it perpendicular to the gas flow path. Then, on carefully withdrawing the card, the layer of powder adhered to the tape was observed at the optical microscope. Images were taken from the vicinity of the free surface, where the bed is diluted, allowing us to discriminate for particle structuring. Typical pictures are shown in fig. 2. In accordance with the difference in their natural cohesiveness, demagnetized steel particles show marked aggregation in the fluidized bed as compared to mostly individual magnetite beads. Right images reveal that this contrasting behavior has consequences on the further aggregation induced by the presence of the horizontal magnetic field. It is seen that the aggregative nature of the steel powder favors the formation of large-scale branched structures. In contrast, structuring in the magnetite bead system mainly consists of the formation of quasilineal chains of just a few beads. This behavior resembles the reported structuring in suspensions of maghemite nanoparticles even though the individual particles were weakly dipolar [5]. In analogy with our observations, magnetic interaction between the nanoparticles was enhanced by their natural aggregation due to enhanced van der Waals attraction.

A striking feature brought to light by fig. 2 is that chainlike structures do not follow the magnetic-field direction as would be expected. In spite that the field is

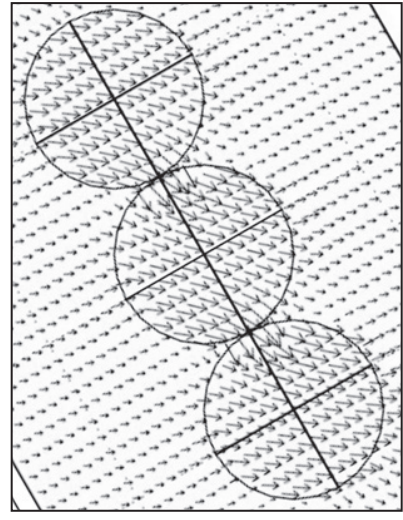

Fig. 3: Magnetic flux density lines obtained by FEM for a $60^{\circ}$ tilted chain of three spherical beads $\left(\chi_{p}=5.33\right)$ subjected to a horizontal magnetic field.

horizontal, chains are seen along quasivertical directions along which the resistance to the vertical gas flow is minimized. Usually, particles in magnetofluidized beds are viewed as point dipoles oriented along the magneticfield lines [3], which would lead us to predict a repulsive force between particles in the quasivertical chains against the experimental evidence offered by fig. 2 . The physical mechanism responsible for the stability of quasivertical chains of beads magnetized by a horizontal field is evidenced in fig. 3, where magnetic flux density field lines from FEM calculations are shown for a $60^{\circ}$ tilted chain of magnetic spheres with susceptibility $\chi_{p}=5.33$ (as obtained for magnetite beads from their measured bulk susceptibility and using Bruggeman mixing rule [8]). Since $\chi_{p}$ is large, the field lines are guided throughout the chain at interparticles contacts, where the field becomes almost normal to the contact surface and its strength is intensified. This distribution yields strong north-to-south poles attraction and thus stabilizes the quasivertical chain.

In the fluidlike bubbling regime the bed does not have a yield stress and the material weight per unit area $W$ is completely balanced by the gas pressure drop $\Delta p$. As the superficial gas velocity $v_{g}$ is decreased there comes a critical point at which particles are jammed in enduring positions and the bed is stabilized. At the jamming threshold enduring particle contacts support part of the structure and thus $\Delta p$ crosses below $W$. This is seen in fig. 4 , where gas pressure drop $\Delta p$ data measured as a function of $v_{g}$ are plotted. While the kinetic arrest is driven by crowding of single particles in the absence of attractive forces, for cohesive particles jamming is driven by the crowding of aggregates, which is likely to occur at higher gas velocities [7]. Accordingly, it is observed that $v_{g}$ at marginal stability $\left(v_{g}=v_{c}\right)$ is larger for the steel powder and is greatly increased when the magnetic field is present and aggregates are larger. Data of $v_{c}$ are plotted in fig. 4 (inset) as a function of the magnetic-field 


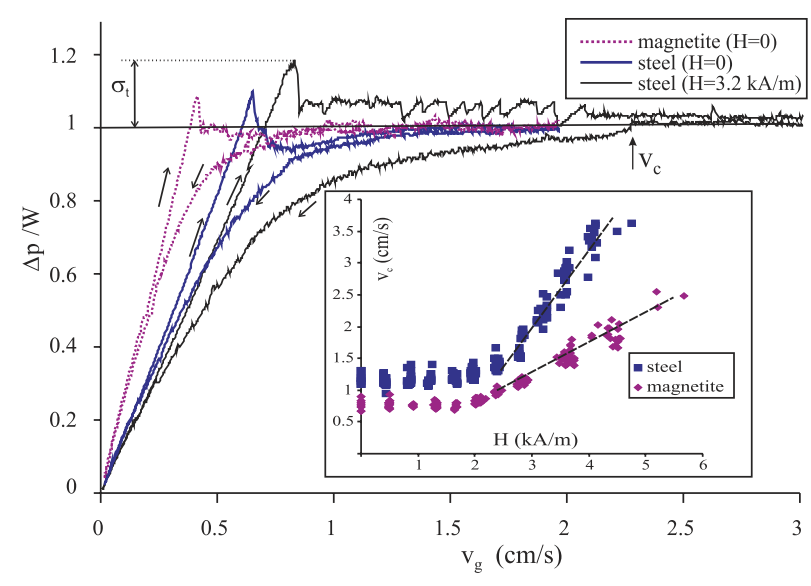

Fig. 4: (Colour on-line) Gas pressure drop across the powder beds as a function of superficial gas velocity during the fluidization-defluidization cycles in the absence and presence of externally imposed magnetic field. The inset shows the gas velocity at marginal stability $v_{c} v s$. the strength of the magnetic field applied.

strength externally applied $H$. It is observed that the field effect becomes noticeable for $H \gtrsim 2 \mathrm{kA} / \mathrm{m}$. At this field strength the interparticle attractive force due to the magnetic interaction prevails against the natural van der Waals force [8]. As $H$ is further increased, $v_{c}$ can be fitted by a linear function $\Delta v_{c} \simeq \alpha H$. For a given field strength, the magnetofluidized bed of steel particles is jammed at higher gas velocities and the slope $\alpha$ is appreciably increased, which can be attributed to the cooperative effect between natural and magnetic aggregation inferred from fig. 2.

Gas pressure drop measurements further allow us to measure the tensile yield stress of the powder bed by subjecting it to a slowly increasing gas velocity. As the gas velocity $v_{g}$ is increased, the bed structure is at first unperturbed and $\Delta p$ increases linearly (see fig. 4). This behavior corresponds to Carman's law for the resistance of porous solids to the passage of gas flow. The larger the porosity the smaller the slope. It can be seen in fig. 4 that the smaller slope is obtained for the steel powder bed settled in the presence of the field as would be expected because of its larger porosity due to enhanced aggregation. At the point of minimum fluidization velocity, $\Delta p$ balances the powder weight per unit area $W$. At this point a powder with zero cohesion would become fluidized, yet the pressure drop across a cohesive powder continues to increase above the minimum fluidization velocity. Above this point the gas flow puts the bed under tension, and as the tension builds up there comes a point at which the powder breaks and the pressure drop falls down to around the weight per unit area $W$ (see fig. 4). Since we are dealing with shallow beds, wall effects are negligible. Thus, the pressure overshoot above $W$ gives an estimate of the tensile yield stress of the powder. As can be observed in fig. 4, the bed settled in the presence of the magnetic

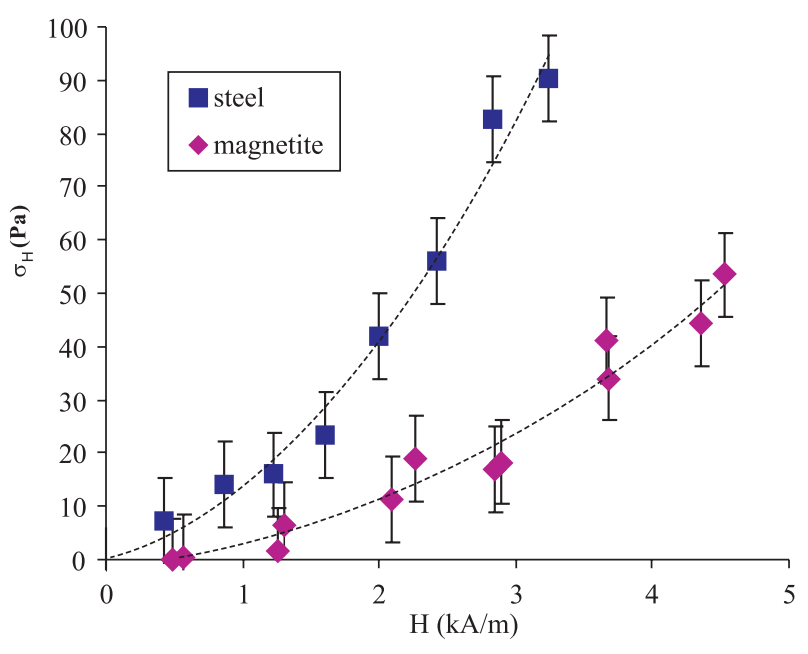

Fig. 5: (Colour on-line) Magnetic cohesion as a function of the strength of the magnetic field for the steel and magnetite powder beds. The dashed lines represent the fitting of the data by a quadratic law.

field has an appreciably larger yield stress. On the other hand, the bed settled in the absence of magnetic field also possesses a yield stress due to the natural cohesiveness of the fine powders used. In order to investigate the yield stress purely induced by the magnetic field, the pressure drop cycle can be reversed from a fluidization state close to marginal stability $\left(v_{g} \lesssim v_{c}\right)$, in which the stabilized bed is unconsolidated and the natural cohesion is negligible [8]. Alternatively, the natural yield stress of the settled bed, measured in the absence of magnetic field, can be subtracted from the total yield stress, measured in the presence of magnetic field. Figure 5 shows data of the yield stress just due to the magnetic field $\left(\sigma_{H}\right)$ obtained in this way. It can be seen that $\sigma_{H}$ can be well fitted by a quadratic law $\sigma_{H}=\beta H^{2}$ as would be expected from the attractive force between soft magnetic particles. Note however that, in spite of the fact that the susceptibilities of both powders are similar, the magnetic cohesion is considerably enhanced for the steel powder. In practice the yield stress is an average of interparticle attractive forces, which is expected to be affected by the structuring of particles during fluidization and transition to jamming. The enhancement of magnetic cohesion is attributable to the coupling between natural and magnetic aggregation.

In conclusion, we have investigated the structure and yield stress of fluidized beds of fine magnetizable particles as affected by a horizontal magnetic field. The magnetic powders used in this work are characterized by having a similar susceptibility but differ in their natural cohesiveness. It has been shown that even though the field is applied in the horizontal direction, it gives rise to a cohesive magnetic force between particles arranged in quasivertical chainlike structures, against the common misconception of instability based on the point dipole 
approximation. According to visual observations the natural cohesiveness of the powder in the absence of field favors the formation of large-scale branched structures in the magnetofluidized bed, which enhances the yield stress and favors stabilization at large values of the gas velocity. Otherwise, if particles in the demagnetized fluidized bed behave individually, mesostructures in the magnetofluidized bed just consist of quasilineal particle chains, stabilization occurs at smaller gas velocities and the magnetic cohesion is smaller. Our work shows therefore that there exists a coupling in magnetofluidized beds between natural cohesiveness and structural stabilization by magnetic forces.

$$
* * *
$$

We acknowledge Spanish Government Agency Ministerio de Ciencia y Tecnologia (FIS2006-03645) and Junta de Andalucia (FQM 421).

\section{REFERENCES}

[1] Rosensweig R. E., Ferrohydrodynamics (Dover Publications, New York) 1997; Hristov J. Y., Powder Technol., 87 (1996) 59.

[2] Siegell J. H., Powder Technol., 57 (1989) 213.

[3] Jones T. B., Electromechanics of Particles (Cambridge University Press, Cambridge) 1995.

[4] Potter D. K. and Stephenson A., Geophys. Res. Lett., 15 (1988) 1097.

[5] Lalatonne Y., Richardi J. and Pileni M. P., Nat. Mater., 3 (2004) 121.

[6] Quintanilla M. A. S., Valverde J. M. and CastelLANos A., J. Stat. Mech.: Theory Exp. (2006) P07015; Quintanilla M. A. S., Valverde J. M., Castellanos A. and Viturro R. E., Phys. Rev. Lett., 87 (2001) 194301.

[7] Valverde J. M., Castellanos A. and Quintanilla M. A. S., Phys. Rev. Lett., 86 (2001) 3020; Castellanos A., Valverde J. M. and Quintanilla M. A. S., Phys. Rev. Lett., 94 (2005) 75501.

[8] Valverde J. M., Espin M. J., Quintanilla M. A. S. and Castellanos A., Phys. Rev. E, 79 (2009) 031306. 